\title{
Vorwort zur dritten Auflage
}

Die zweite Auflage des Buches hat sich beim Unterricht im Laboratium gut bewährt. Deshalb ist die vorliegende dritte Auflage Im wesentlichen unverändert geblieben. Das dürfte auch den alten Freunden des Buches, die es beim praktischen Anfänger-Unterricht in der Chemie zugrunde legten, nach Wunsch sein.

Die vorgenommenen Veränderungen betreffen notwendige Verbesserungen, auf die ich vielfach von den Fachgenossen aufmerksam gemacht wurde, wofür ich auch an dieser Nitelle meinen besten Dank aurssprechen möchte. Auch künftig werde ich weiteren Anregungen zu Verbesserungen gern und dankbar entgegensehen.

Von den Veränderungen seien hier nur einige erwähnt: Die Bezeichnung der Löslichkeit der Stoffe ist in der neuen Auflage überall nach einheitlicher Zählung ( $\mathrm{g}$ substanz in $100 \mathrm{~g}$ Lösungsmittel) durchgeführt worden. Die Löslichkeit einiger weiterer Stoffe, die analytisch zur Beurteilung der Empfindlichkeit der Fällungsreaktionen bedeutungsvoll sind, wurde neu aufgenommen. Ebenso hat der mikrochemische Nachweis wägbarer Mengen Natrium als Natriumuranylacetat und die Aufschließung der silikate nach P. JANNASCHs Borsäuremethode Aufnahme gefunden.

Das periodische System der Grundstoffe ist nach der neusten Entwicklung der Lehre von den chemischen Elementen umgeändert worden, auf deren Wesen und Bedeutung freilich im Rahmen dieses Buches nicht eingegangen werden konnte. Darüber möge in K. FAJans „Radioaktivität und neuste Entwicklung der Lehre von den chemischen Elementen,“ Sammlung Vieweg, Tagesfragen, Heft 45, 1919 und auch in L. GraETz ,.Die Atomtheorie in ihrer neuesten Entwicklung,“ Stuttgart, Verlag J. Engelhorns Nachf., 1918 nachgelesen werden.

Bei den Änderungen und beim Lesen der Korrektur hat mir Herr Dr. Gustay Oelbermann wertvolle Hilfe geleistet, was ich auch hier mit bestem Dank anerkennen möchte.

Heidelberg, im Juni 1920.

\section{E. Knoevenagel}

